\title{
ESTIMATIVA DA ÁREA FOLIAR DE PLANTAS DANINHAS: Brachiaria decumbens Stapf. e Brachiaria brizantha (Hochst.) Stapf ${ }^{1}$
}

\author{
SILVANO BIANCO², RODRIGO A. BRENDOLAN ${ }^{3}$, PEDRO L. da C. A. ALVES ${ }^{4}$ e ROBINSON A. PITELLI
}

\section{RESUMO}

Com o objetivo de obter uma equação que, através de parâmetros lineares dimensionais das folhas, permita a estimativa da área foliar de Brachiaria decumbens Stapf. e Brachiaria brizantha (Hochst.) Stapf., estudaram-se correlações entre a área foliar real (Sf) e parâmetros dimensionais do limbo foliar, como o comprimento ao longo da nervura principal $(\mathbf{C})$ e a largura máxima $(\mathbf{L})$, perpendicular à nervura principal. Todas as equações, exponenciais, geométricas ou lineares simples, permitiram boas estimativas da área foliar. Do ponto de vista prático, sugere-se optar pela equação linear simples envolvendo $\mathrm{o}$ produto $\mathbf{C} \quad \mathbf{x} \quad \mathbf{L}$, considerando o coeficiente linear igual a zero. Desse modo, a estimativa da área foliar de $B$. decumbens pode ser feita pela fórmula Sf $=\mathbf{0 , 9 8 1 0} \times(\mathbf{C} \times \mathbf{L})$, ou seja, $98,10 \%$ do produto entre o comprimento ao longo da nervura principal e a largura máxima, enquanto que, para a $B$. brizantha a estimativa da área foliar pode ser feita pela fórmula $\mathbf{S F}=\mathbf{0 , 7 4 6 8} \times(\mathbf{C} \times \mathbf{L})$, ou seja $74,68 \%$ do produto entre o comprimento ao longo da nervura principal e a largura máxima da folha.

Palavras-chave: Fisiologia vegetal, gramíneas.

\section{ABSTRACT}

\section{Leaf area estimative in weeds Brachiaria decumbens Stapf and Brachiaria brizantha (Hochst.) Stapf}

In order to obtain equations which make feasible the leaf area estiomate from linear measures of the leaf blade, correlation studies were done involving the real leaf blade area and main vein leaf lenght $(\mathbf{C})$, maximum leaf width (L) and $\mathbf{C} * \mathbf{L}$. All the equations, linear, geometric and exponential, provide good leaf area estimate for both species. In the practical sense, it is suggested the use of the simple linear equation of the regression model using the $\mathbf{C} * \mathbf{L}$ parameter and taking the linear coefficient equal zero. Then, the Brachiaria decumbens leaf area can be estimate using the equation $\mathbf{S f}=\mathbf{0 , 9 8 1 0}\left(\mathbf{C}^{*} \mathbf{L}\right)$ and the Brachiaria brizantha by using the equation Sf $=0,7468 *(C * L)$.

Key words: Weed physiology, grass weeds.

\section{INTRODUÇ̃̃O}

Brachiaria decumbens Stapf. e Brachiaria brizantha (Hochst.) Stapf. são espécies introduzidas da África do Sul como excelentes forrageiras perenes apresentando grande produção de massa foliar de boa qualidade e boa resistência ao pastoreio intensivo e ao pisoteio, especialmente em vastas regiões do Brasil Central.

\footnotetext{
${ }^{1}$ Recebido para publicação em 10/03/99 e na forma revisada em 18/05/99.

${ }^{2}$ Prof $^{\circ}$ Dr., FCAV/UNESP. CEP: 14.870.000, Jaboticabal/SP, Brasil.

${ }^{3}$ Acadêmico em Biologia, União das Faculdades Barão de Mauá, Ribeirão Preto/SP.

${ }^{4}$ Prof ${ }^{\circ}$ Dr., FCAV/UNESP. CEP: 14.870.000, Jaboticabal/SP, Brasil.

${ }^{5}$ Prof ${ }^{\mathrm{o}}$ Titular, FCAV/UNESP. CEP: 14.870-000, Jaboticabal/SP, Brasil.
} 
Devido o caráter exótico e elevada agressividade, estas duas espécies passaram a invadir áreas de agricultura, fruticultura e reflorestamento, tornando-se importante plantas daninhas, especialmente Brachiaria decumbens. Kissmann (1997) comenta que sérios problemas com Brachiaria decumbens ocorrem em lavouras de soja na Região Centro-Oeste do Brasil e em lavouras de cana-de-açúcar no Estado de São Paulo. Além da competição imposta, esta planta tem reduzido fortemente a vida útil de canaviais infestados, para um máximo de 2 ou 3 cortes. Em pomares infestados, o desenvolvimento de mudas de citrus é retardado, sugerindo efeito alelopático.

$\mathrm{Na}$ cultura do eucalipto, as braquiárias tornaram-se extremamente problemáticas, especialmente pelo fato de que a expansão dessa cultura ocorreu em áreas anteriormente ocupadas por pastagens (Marchi et al., 1995).

Considerando-se a importância dessas plantas, há grande necessidade de estudos básicos envolvendo aspectos relacionados à reprodução, crescimento, desenvolvimento, exigências em nutrientes, respostas aos sistemas de controle e outros. Na maioria desses estudos, o conhecimento da área foliar é fundamental, pois é talvez o mais importante parâmetro na avaliação do crescimento vegetal. Existem vários métodos para se medir a área foliar, a maioria com boa precisão. Marshall (1968) os classificou em destrutivos e não destrutivos, diretos ou indiretos. A importância de se realizar um método não destrutivo, permite acompanhar o crescimento e a expansão foliar da mesma planta até o final do ciclo ou do ensaio, além de ser rápido e relativamente preciso. Assim, prefere-se a estimativa utilizando-se parâmetros dimensionais de folhas que apresentam boas correlações com a superfície foliar. Um dos métodos não destrutivos mais utilizados é a estimativa da área foliar por meio de equações de regressão entre a área foliar real (Sf) e parâmetros dimensionais lineares das folhas. Este método já foi utilizado com sucesso para inúmeras plantas cultivadas e plantas daninhas, como Euphorbia heterophylla $\mathrm{L}$. (Ribeiro et al., 1977); Wissadula subpeltata
(Kuntze) Fries (Bianco et al., 1983); Ipomoea aristolochiaefolia (H.B.K.) Don e I. acuminata Roem et Sch (Vellini, et al., 1983), Senna obtusifolia (L.) Irwin \& Barneby. (Peressin et al., 1984), Amaranthus retroflexus L. (Bianco et al., 1995), Richardia brasiliensis (Gomez) (Rosseto et al., 1997), entre outras.

O presente trabalho tem por objetivo estudar equações para estimativa da área foliar de Brachiaria decumbens Stapf e Brachiaria brizantha (Hochst.) Stapf. a partir de dimensões lineares de seus limbos foliares.

\section{MATERIAL E MÉTODOS}

Foram coletados 200 limbos foliares de $B$. decumbens e de $B$. brizantha sujeitos às mais diversas condições ecológicas que as espécies são susceptíveis de ocorrer como infestante, considerando-se toda as folhas das plantas, desde que não apresentassem deformações oriundas de fatores externos como pragas, moléstias e granizo. No laboratório, foram determinados o comprimento do limbo foliar ao longo da nervura principal $(\mathbf{C})$ e a largura máxima do limbo foliar (L) perpendicular à nervura principal. A seguir, as folhas foram desenhadas em papel homogêneo e suas áreas foliares reais ( $\mathbf{S f}$ ) determinadas através do aparelho "Portable Area Meter" Licor Mod. L1 - 3000 .

Para escolha de uma equação que possa representar a área foliar procederam-se estudos de regressão com as equações linear $\mathbf{Y}=\mathbf{a}+\mathbf{b x}$; geométrica $\mathbf{Y}=\mathbf{a} \mathbf{x}^{\mathbf{b}}$ e exponencial $\mathbf{Y}=\mathbf{a} \mathbf{b}^{\mathbf{x}}$. $\mathrm{O}$ valor $\mathbf{Y}$ estima a área foliar do limbo foliar em função de $\mathbf{X}$, cujos valores podem ser $\mathbf{o}$ comprimento (C), a largura (L) ou o produto $(\mathbf{C} \times \mathbf{L})$. No caso de $\mathbf{X}$ igual ao $(\mathbf{C} \times \mathbf{L})$, estimouse também a equação linear passando pela origem, o que praticamente significa supor que a área é proporcional a um retângulo $(\mathbf{C} \mathbf{x} \mathbf{L})$. A melhor equação é a que apresenta a menor soma de quadrados do resíduo na escala real (sem transformação). 


\section{RESULTADOS E DISCUSSÃO}

As equações obtidas com os respectivos coeficientes de determinação estão nas Tabelas 1 e 2. Pode-se observar que todas as equações de regressão relacionando a área foliar real e os parâmetros dimensionais de comprimento, largura máxima e do produto entre o comprimento e a largura máxima podem permitir boas estimativas das áreas do limbo foliar destas espécies. No entanto, as melhores estimativas foram obtidas com o produto do comprimento pela largura, uma vez que os desvios, devidos a fatores não controlados, foram sensivelmente menores e o coeficiente de determinação atingiu 0,9320 e 0,8725 , respectivamente para $B$. decumbens e $B$. brizantha, sugerindo que $93,20 \%$ e $87,25 \%$ das variações totais observadas podem ser explicados pelas equações determinadas.

TABELA 1. Equações de regressão entre a área foliar real (Sf) e parâmetros dimensionais lineares do limbo foliar de Brachiaria decumbens Stapf. FCAV - UNESP. Jaboticabal/SP. 1999.

\begin{tabular}{lccccc}
\hline $\mathrm{X}^{(1)}$ & forma & $\begin{array}{c}\text { Coeficiente } \\
\text { de } \\
\text { Determinação }\end{array}$ & GL & $\begin{array}{c}\text { S. Q. resíduo } \\
\text { (sem transformação) }\end{array}$ & Equação $^{(2)}$ \\
\hline $\mathrm{L}$ & linear & 0,6885 & 198 & 3731,02 & $\mathbf{S f}=-6,5706+25,3941 \mathrm{~L}$ \\
$\mathrm{CL}$ & linear & 0,9320 & 198 & 932,06 & $\mathbf{S f}=2,2241+0,8718 \mathrm{CL}$ \\
$\mathrm{CL}(0,0)^{\#}$ & linear & 0,9320 & 199 & 1040,61 & $\mathbf{S f}=0,9810 \times \mathrm{CL}$ \\
$\mathrm{C}$ & geométrica & 0,8812 & 198 & 1972,79 & $\mathbf{S f}=0,6146 \times \mathrm{C}^{1,1535}$ \\
$\mathrm{~L}$ & geométrica & 0,7258 & 198 & 3891,79 & $\mathbf{S f}=18,1932 \times \mathrm{L}^{1,5822}$ \\
$\mathrm{C}$ & exponencial & 0,7258 & 198 & 3227,84 & $\mathbf{S f}=5,0287 \times 1,0680^{\mathrm{C}}$ \\
$\mathrm{L}$ & exponencial & 0,7034 & 198 & 4207,61 & $\mathbf{S f}=3,3318 \times 5,3450^{\mathrm{L}}$ \\
\hline
\end{tabular}

(1) parâmetros dimensionais lineares; (2) área foliar estimada

\# não há significância entre as duas equações lineares envolvendo o produto C x L.

TABELA 2. Equações de regressão entre a área foliar real (Sf) e parâmetros dimensionais lineares do limbo foliar de Brachiaria brizantha (Hochst.) Stapf. FCAV - UNESP. Jaboticabal/SP. 1999.

\begin{tabular}{|c|c|c|c|c|c|}
\hline$X^{(1)}$ & forma & $\begin{array}{l}\text { Coeficiente } \\
\text { de } \\
\text { Determinação }\end{array}$ & GL & $\begin{array}{c}\text { S. Q. resíduo } \\
\text { (sem transformação) }\end{array}$ & Equação $^{(2)}$ \\
\hline $\mathrm{C}$ & linear & 0,8131 & 198 & 1476,75 & $\mathbf{S f}=-5,5231+1,3906 \mathrm{C}$ \\
\hline $\mathrm{L}$ & linear & 0,6231 & 198 & 4471,54 & $\mathbf{S f}=-0,8078+18,4465 \mathrm{~L}$ \\
\hline $\mathrm{CL}^{\#}$ & linear & 0,8725 & 198 & 1745,47 & $\mathbf{S f}=3,8459+0,6462 \mathrm{CL}$ \\
\hline $\mathrm{CL}(0,0)^{\#}$ & linear & 0,8725 & 199 & 1887,06 & $0,7468 \times C L$ \\
\hline $\mathrm{C}$ & geométrica & 0,8323 & 198 & 2538,94 & $\mathbf{S f}=0,4357 \times C^{1,3047}$ \\
\hline $\mathrm{L}$ & geométrica & 0,6344 & 198 & 4529,22 & $\mathbf{S f}=16,6777 \times \mathrm{L}^{1,1270}$ \\
\hline $\mathrm{C}$ & exponencial & 0,6344 & 198 & 2781,39 & $\mathbf{S f}=6,7178 \times 1,0599^{C}$ \\
\hline $\mathrm{L}$ & exponencial & 0,6283 & 198 & 4601,52 & $\mathbf{s f}=8,1233 \times 2,1736^{\mathrm{L}}$ \\
\hline
\end{tabular}


Do ponto de vista prático, dentre as melhores equações, a de fácil manuseio é aquela que envolve $\mathrm{o}$ produto do comprimento pela largura máxima do limbo foliar. Ainda observa-se que forçando a passagem da reta pela origem, não houve aumento sensível no valor da soma de quadrados de resíduos. A relação de equações para estimativa da área foliar pode ser visualizada na Figura 1. Sugere-se, portanto, que estimativa relativamente simples e precisa da área do limbo foliar de $B$. decumbens, pode ser obtida pela equação $\mathbf{S f}=\mathbf{0 , 9 8 1 0} \times(\mathbf{C} \times$ L), ou seja, $98,10 \%$ do produto entre o comprimento e a largura máxima do limbo foliar. Para $B$. brizantha, esta estimativa pode ser obtida pela equação $\mathbf{Y}=\mathbf{0 , 7 4 8 0} \times(\mathbf{C} \times \mathbf{L})$, ou seja, $74,80 \%$ do produto entre o comprimento e a largura máxima do limbo foliar.

Todas as equações obtidas no presente trabalho podem ser utilizadas para estimar a área foliar de Brachiaria decumbens e Brachiaria brizantha. Porém, as estimativas mais precisas serão obtidas utilizando-se a equação Sf $=0,9810 \times(C \times L)$ e $\mathbf{S f}=\mathbf{0 , 7 4 6 8} \times(C \times L)$ passando pela origem, com um coeficiente de determinação da ordem de 0,9320 e 0,8725 , respectivamente.
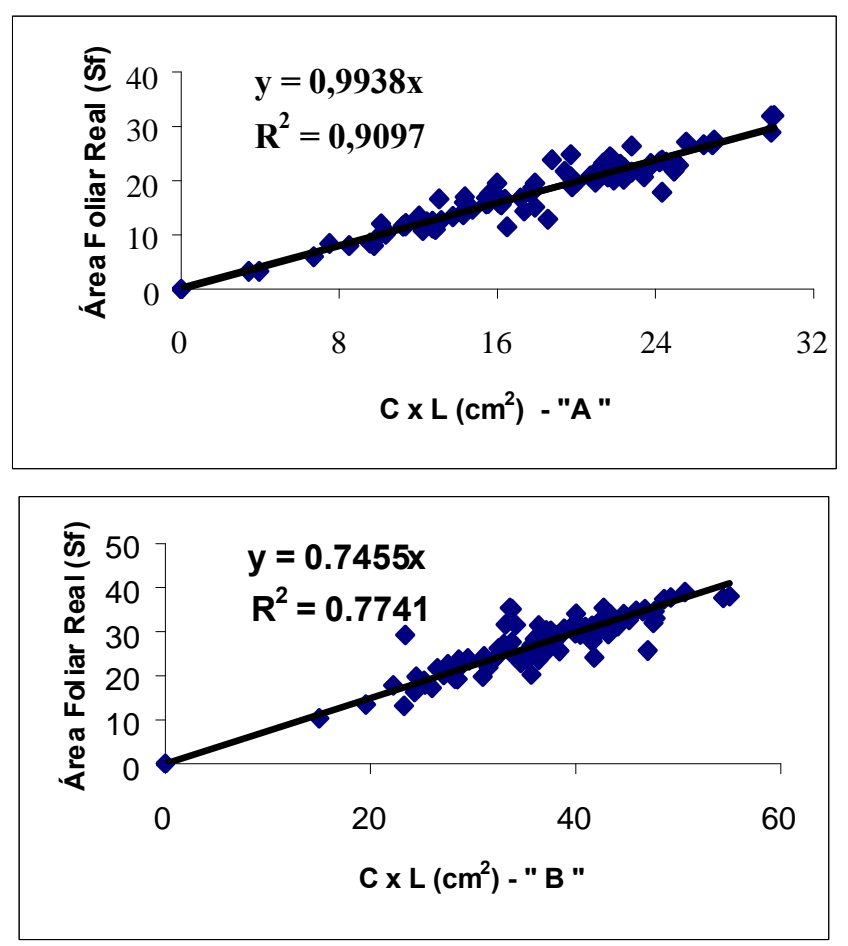

FIGURA 1. Valores observados do produto entre comprimento e largura máxima $(\mathbf{C} \times \mathbf{L})$ e a área foliar de Brachiaria decumbens Stapf. "A" e Brachiaria brizantha (Hochst. Stapf. "B" (Sf) e representação esquemática das equações de regressão obtidas.

\section{LITERATURA CITADA}

BIANCO, S.; PITELLI, R. A. \& PERECIN, D. Métodos para estimativa da área foliar de plantas daninhas. 2. Wissadula subpeltata
(Kuntze) Fries. Planta Daninha, v.6, n.1, p.21- 24, 1983.

BIANCO, S.; PITELLI, R.A.; PAVANI, M. C. M. D. \& SILVA, R. C. Estimativa de área foliar de plantas daninhas. XIII - 
Amaranthus retroflexus

L. Ecossistema vol. 20, p. 5-9, 1995.

KISSMANN, K. G.; GROTH, D. Plantas Infestantes e Nocivas. Tomo I. $2^{\text {a }}$ Edição. São Paulo. BASF Brasileira, 1997. 824p.

MARCHI,S. R.; PITELLI, R.A.; BEZUTTE, A.J.; CORRADINE, L.; ALVARENGA, S. F. Efeito de períodos de convivência e de controle das plantas daninhas na cultura de Eucalyptus grandis. In: SEMINÁRIO SOBRE CULTIVO MÍNIMO DO SOLO EM FLORESTAS. 1º, Curitiba/PR, 1995. Anais, p. 122-133.

MARSHALL, J. K. Methods of leaf area measurement of large and small leaf samples. Photosynthetica, Praha, v. 2, p. 41-47, 1968.

PERESSIN, V. A.; PITELLI, R. A. \& PERECIN, D. Métodos para estimativa da área foliar de plantas daninhas. 4. Cassia tora L. Planta Daninha, v.7, n.2, p. 48-52, 1984.

RIBEIRO, C, J.; PITELLI, R. A. \& PERECIN, D. Comparação de métodos para estimativa da área foliar de Euphorbia heterophylla L. In: CONGRESSO NACIONAL DE BOTÂNICA, 28 ${ }^{\circ}$, Belo Horizonte/MG, 1977. Resumos, s/p.

ROSSETO, R. R.; PITELLI, R. L. C. M.; PITELLI, R. A. Estimativa da área foliar de plantas daninhas: Poaia-Branca. Planta Daninha, v.15, n.1, p. 25-29, 1997.

VELLINI, E. D.; DOMINGUES, E. P.; PITELLI, R. A. \& PERECIN, D. Estimativa da área foliar em plantas daninhas. 3. Ipomoea acuminata Roem et Sch. e Ipomoea aristolochiaefolia (H.B.K.) Don. In: CONGRESSO BRASILEIRO DE INICIAÇÃO CIENTÍFICA EM CIÊNCIAS AGRÁRIAS, 3으. Florianópolis/SC, 1983. Resumos, p. 23. 